\title{
COVID-19 guidance for triage of operations for thoracic malignancies: A consensus statement from Thoracic Surgery Outcomes Research Network
}

Thoracic Surgery Outcomes Research Network, Inc*

\section{ABSTRACT}

The extraordinary demands of managing the COVID-19 pandemic has disrupted the world's ability to care for patients with thoracic malignancies. As a hospital's COVID19 population increases and hospital resources are depleted, the ability to provide surgical care is progressively restricted, forcing surgeons to prioritize among their cancer populations. Representatives from multiple cancer, surgical, and research organizations have come together to provide a guide for triaging patients with thoracic malignancies as the impact of COVID-19 evolves as each hospital. (J Thorac Cardiovasc Surg 2020;160:601-5)

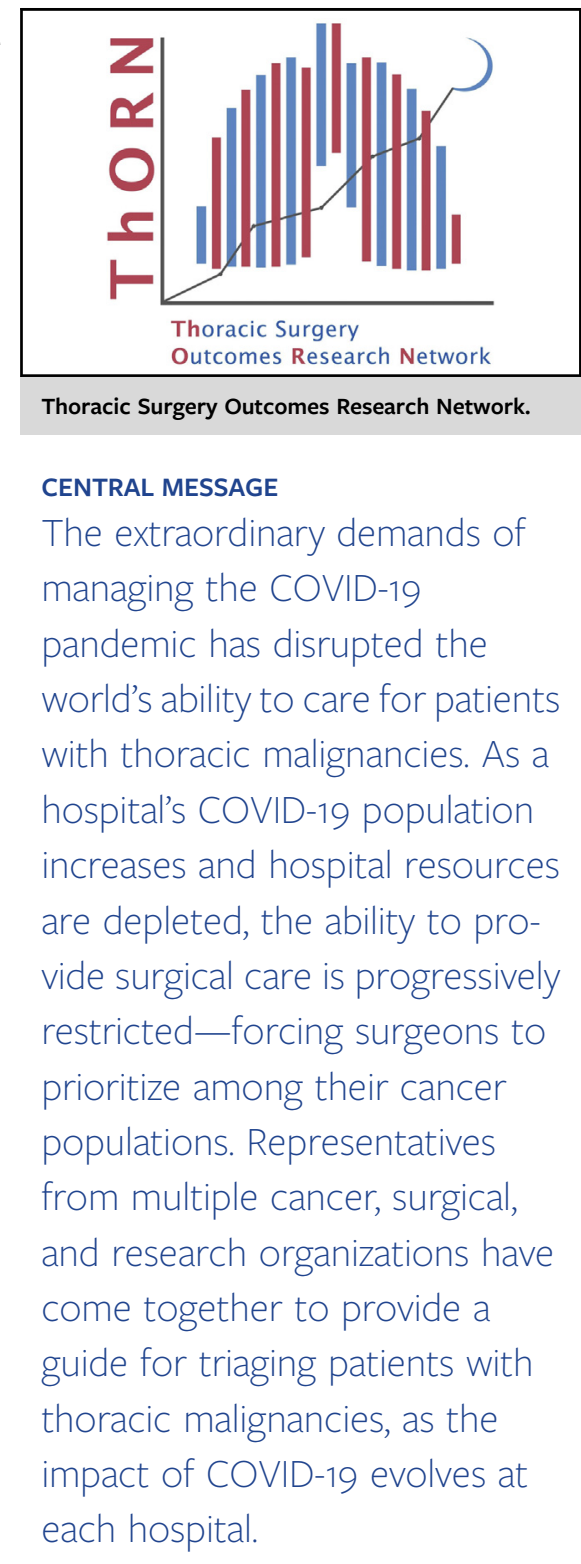

The American Association for Thoracic Surgery and The Society of Thoracic Surgeons support this document.

This article has been copublished in The Journal of Thoracic and Cardiovascular Surgery and The Annals of Thoracic Surgery.

The American Association for Thoracic Surgery requests that this article be cited as: Thoracic Surgery Outcomes Research Network, Inc. COVID-19 Guidance for triage of operations for thoracic malignancies: a consensus statement from Thoracic Surgery Outcomes Research Network. J Thorac Cardiovasc Surg. 2020;160:601-5.

* A complete list of the authors in the Thoracic Surgery Outcomes Research Network, Inc, group appears at the end of this article.
Received for publication March 23, 2020; revisions received March 24, 2020, accepted for publication March 24, 2020; available ahead of print April 9, 2020. Address for reprints: Daniel J. Boffa, MD, PO Box 208062, New Haven, CT 065208062 (E-mail: daniel.boffa@yale.edu).

J Thorac Cardiovasc Surg 2020;160:601-5 $0022-5223 / \$ 36.00$

Copyright (C) 2020 by The Society of Thoracic Surgeons and the American Association for Thoracic Surgery. Published by Elsevier Inc.

https://doi.org/10.1016/j.jtcvs.2020.03.061 
The COVID-19 pandemic has forced hospitals to progressively reduce surgical volumes to both minimize disease transmission within the hospital and to preserve human and personal protective equipment and other resources needed to care for COVID-19 patients. In response, many hospitals have abruptly reduced or eliminated elective operations. As the COVID-19 burden on a hospital increases, procedures that improve survival may similarly have to be reduced or eliminated (ie, semielective, urgent, and perhaps some emergent operations).

For some cancer patients, surgery may be delayed for months or even years without negative consequences. In other scenarios, however, failure to perform an indicated cancer surgery in a timely fashion may have long-term implications on a patient's survivorship or significant permanent deficits in their quality of life. Therefore, cancer patients and the oncology teams that treat them are likely to face difficult decisions between suboptimal management strategies.

Thoracic oncology decisions are further complicated by the fact most of the patients with lung, esophageal, and other thoracic malignancies would be considered to be a high-risk group for poor outcomes with COVID-19 (advanced age, emphysema, and heart disease). Further, the indicated therapeutic procedures can both impair lung function (ie, lung isolation, removal of lung tissue) and expose clinical teams to aerosolized viral load (bronchoscopy, double-lumen endotracheal tube placement, airway surgery, laparoscopy and possibly lung surgery particularly with parenchymal lung leaks). We have assembled a document to offer guidance intended to facilitate these difficult decisions when caring for patients with thoracic malignancies during the COVID-19 pandemic (Table 1). ${ }^{1-10}$

\section{ASSUMPTIONS}

Much of the impact, timeline, duration, risks, and ultimate recovery from the COVID-19 pandemic remain unknown. In an effort to give context to this triage guide, several assumptions have been made:

- The risk of nosocomial infection (patients and clinicians infected while in hospital $)^{11-15}$ and competition for resources (surgical and medical patients) will increase in proportion to the prevalence of hospitalized COVID19 patients.

- The duration of restriction on elective surgery will last approximately 3 months.

- Each facility's progression through the phases of care restriction will be variable, but surgeons should be prepared for rapid changes in hospital status (ie, consider what eligible operations could or should be performed as soon as possible).

- Surgical leadership are provided with daily updates regarding a hospital's COVID-19 population and resource status.

\section{PROCESS OF PRIORITY STATUS DETERMINATION FOR INDIVIDUAL PATIENTS}

There are nuances to each patient's management approach, such as proceeding with surgery, delaying surgery, or pursuing alternative treatment, that will impact risk tolerance for both patient and surgeon. Ideally, when traditional cancer treatment is not logistically feasible, a patient's care plan will be made with input from a group of clinicians with expertise in thoracic malignancies, such as a case conference or tumor board. We encourage the use of this multidisciplinary strategy as guidance as appropriate for each individual hospital or clinic setting. Several considerations may cause a group's consensus approach to differ from what is proposed in Table 1:

- The risk of delay may not be specifically captured by the outlined descriptors (ie, tumor may have aggressive growth kinetics or histology).

- Resource limitations (clinicians, supplies, facilities) affecting surgical, medical and radiation oncology departments may pose heterogeneous restrictions from hospital to hospital.

- Clinicians will need to keep in mind the important concept of social distancing in modifying management to limit the number of visits to the hospital for any reason.

In addition, because the duration of surgical volume restriction is unknown (3 months is presumed), patients who are delayed or deferred should be tracked (ie, a patient registry or database). Considerations for the database should include the following:

- Indication if reassessment during the period of delay could influence care plan (ie, follow-up computed tomographic scan). This should be extremely selective, because access to imaging will likely be increasingly restricted with increased COVID-19 prevalence.

- An indication of case priority (ie, first group, second group, third group) for rescheduling when restrictions are lifted to best care for patients whose survival may be most impacted by additional delay.

- Alternative treatment strategies used in lieu of surgical resection (ie, systemic chemotherapy, stereotactic body radiotherapy, or other ablative strategies, palliative stent placement, etc) should also be tracked.

\section{DISCLAIMERS}

This guidance document is meant to serve patients based on estimates of risk for average patients (in terms of tumor behavior, patient health, hospital resource availability) associated with each strategy.

- These should not be considered rigid guidelines. This guide is not intended to supplant clinical judgment or the development of consensus regarding institutional 
TABLE 1. Guidance for the triage of patients with thoracic malignancies

Phase I

- Few COVID 19 patients in hospital

- Hospital resources intact (eg, ICU beds, ventilators, clinicians, PPE)

- COVID-19 trajectory not in rapid escalation phase

Compass Statement: Surgery restricted to patients whose survivorship is likely to be compromised by surgical delay of 3 months

Surgery performed as soon as feasible

- Solid or predominantly solid (>50\%) lung cancer or presumed lung cancer $\geq 2 \mathrm{~cm}$, clinical node negative

- Node-positive lung cancer

- Postinduction therapy cancer

- Esophageal cancer T1b or greater

- Chest wall tumors of high malignant potential

- Stenting for obstructing esophageal tumor

- Staging to start treatment (EBUS, mediastinoscopy, diagnostic VATS for pleural dissemination $) \S$

- Symptomatic mediastinal tumors-diagnosis not amenable to needle biopsy

- Patients enrolled in therapeutic clinical trials
Surgery deferred (estimate 3 months)*

- Predominantly ground glass $(<50 \%$ solid $)$ nodules or cancers

- Solid nodule or lung cancer $<2 \mathrm{~cm}$

- Indolent histology (eg, carcinoid, slowly enlarging nodule)

- Thymoma (nonbulky, asymptomatic)

- Pulmonary oligometastases, unless clinically necessary for pressing therapeutic or diagnostic indications (ie, surgery will impact treatment)

- Patients likely to require prolonged ICU needs (ie, particularly high-risk patients)

- Tracheal resection (unless aggressive histology)

- Bronchoscopy

- Upper endoscopy $\|$

- Tracheostomy\|
Alternative treatment considered

- Endoscopic therapy for early-stage esophageal cancer (stage $\mathrm{T} 1 \mathrm{a} / \mathrm{b}$ superficial)

- If eligible for adjuvant therapy, then consider neoadjuvant therapy (eg, chemotherapy for 5cm lung cancer) $\dagger^{\dagger} \dagger$

- Stereotactic ablative radiotherapy $\mathbb{1}$

- Ablation (eg, cryotherapy, radiofrequency ablation)

- Stent for obstructing cancers then treat with chemoradiation

- Debulking $\|$ (endobronchial tumor) only in circumstance where alternative therapy is not an option due to increased risk of aerosolization (eg, stridor postobstructive pneumonia not responsive to antibiotics)

- Nonsurgical staging (EBUS, imaging, interventional radiology biopsy) $\mid$

- Monitor patients after their neoadjuvant for "local only failure" (ie, salvage surgery)\#

\section{Phase II}

- Many COVID 19 patients

- Resources limited (eg, ICU beds, ventilators, clinicians, PPE)

- COVID trajectory within hospital in rapidly escalating phase

Compass Statement: Surgery restricted to patients likely to have survivorship compromised if surgery not performed within the next few days

Surgery performed as soon as feasible

- Perforated cancer of esophagus-not septic

- Tumor-associated infection-compromising, but not septic (eg, debulking for postobstructive pneumonia)

- Tumor associated with hemorrhage, not amenable to nonsurgical treatment

- Management of surgical complications (hemothorax, empyema, infected mesh) in a hemodynamically stable patient

Surgery deferred (estimate 3 months)

- All thoracic procedures typically scheduled as routine/elective

Alternative treatment recommended $* *$

\section{Phase III}

- Hospital resources are predominately routed to COVID 19 patients

- Resources critically limited/exhausted

Compass Statement: Surgery restricted to patients likely to have survivorship compromised if surgery not performed within next few hours

Surgery performed as soon as feasible Surgery deferred (estimate 3 months) Alternative treatment at alternate facility

- Perforated cancer of esophagus — septic patient

- All nonemergent operations

- See above

- Threatened airway

- Transfer patient to hospital that is in Phase I

- If eligible for adjuvant therapy, then give neoadjuvant therapy

- Stereotactic ablative radiotherapy for

- Ablation (eg, cryotherapy, radiofrequency ablation)

- Reconsider neoadjuvant as definitive chemoradiation, and monitor patients for "local only failure" (ie, salvage surgery) 
TABLE 1. Continued

- Tumor associated sepsis

- Management of surgical complications-

unstable patient (active bleeding not

amenable to nonsurgical management,

dehiscence of airway, anastomotic leak with

sepsis)

Table 1 defines 3 phases of hospital status based on (A) the prevalence of COVID-19 patients within the hospital, (B) availability of hospital resources, and (C) the rate of change (in terms of increasing prevalence of infections and resource depletion). Because there are unique considerations for individual patients, each phase is accompanied by a "compass statement" that is meant to give additional direction to navigate volume restriction based on perceived risk to patients and hospital staff. For each phase, surgeons should operate for recommended scenarios (first column) but also for recommended scenarios from all higher phases (ie, appropriate operations during Phase II, include first column under both Phase II or Phase III). There are very limited data to inform many key decisions. The data and references in this section are meant to serve as an estimate of effect size, using the largest data sets available. They are not complete and, therefore, should not be used as definitive data but are only suggestive of the magnitude of effect. ICU, Intensive care unit; $P P E$, personal protective equipment; $E B U S$, endobronchial ultrasound; VATS, video-assisted thoracoscopic surgery. *A study from the National Cancer Database suggests that the interval between diagnosis and surgery (ie, time-to-treat) for stage I lung greater than 8 weeks is associated a reduction in 5 -year survival $(54.8 \%$ vs $48.7 \%, P>.001)$. ${ }^{1}$ For stage III lung cancer patients, a delay of greater than 3 months between neoadjuvant therapy and surgery was associated with shorter median survival ( 33.2 months vs 39.8 months, $P=.03) .{ }^{2}$ Smaller institutional studies have not revealed a clear association between the diagnosis-to-treatment interval and long-term outcomes in patients with esophageal cancer. $^{3}$ A delay of greater than 8 weeks between neoadjuvant therapy and surgery for esophageal cancer is not associated with decrement in long-term survival. ${ }^{4} \dagger$ Availability of alternative treatments may vary across health systems and over time. The decision to pursue alternative treatment must balance risk of deferring alternative treatment (chemotherapy and radiotherapy) with risk of exposure of both patients and staff to COVID-19 infection. In Phase I, alternative treatments predominately considered in patients felt to be harmed by delay are listed (ie, the first column of table). $\ddagger$ At the time of writing, the risk of death with COVID-19 infection is felt to be higher among patients receiving chemotherapy, but the data are incredibly limited (18 cancer patients in China). ${ }^{5}$ §Although the accuracy of the clinical staging examination may be enhanced by invasive staging procedures, the magnitude of survival benefit from superior staging may be considered by some to be modest. In the setting of strained resources and potential exposure risk to clinical staff from staging procedures (bronchoscopy and mediastinoscopy), treating a patient based exclusively on a noninvasive staging evaluation (ie, imaging alone) is reasonable. $\|$ These procedures are currently felt to be associated with a particularly high potential to disseminate COVID-19. They should be done selectively and ideally in patients who have been screened for active COVID-19 infection. $\uparrow$ There are incomplete data comparing surgery to stereotactic ablative radiotherapy for early-stage lung cancer in patients eligible for surgery. Observational data, which is likely biased with patients who were not surgical candidates, suggests a modest survival advantage of surgery $(5 \%$ - $15 \%$ higher 5 -year survival) ${ }^{6-8}$ \#Among presumably highly selected patients, salvage resection has been associated with reasonable survivorship after definitive nonsurgical therapy for esophageal cancer, particularly if the patient has had a good response by imaging. ${ }^{9,10} * *$ Recommended for patients in whom a delay would likely compromise survival (ie, first column from Phase I section).

approaches to cancer treatment. There is a great deal of uncertainty around this evolving pandemic and information may change rapidly.

- Critical portions of the transition are not addressed. In reality, there is likely to be a Phase "1a," "1b," "1c," where only fraction of the priority cancer patients may have access to surgery. Clinicians may have to further restrict of surgery, likely across specialties (ie, colon cancer, breast, hepatobiliary) based on the perceived magnitude of risk of delay and over shorter time periods (ie, impact of 8-week delay, then 4 weeks, etc).

- Preoperative evaluation is likely to be impacted (ie, pulmonary function testing), and preoperative screening for COVID-19 is evolving (survey for symptoms, temperature assessment, possible selected testing for COVID19 where available).

- It is possible that the strategies outlined in this document could be replaced as our understanding of unique challenges that COVID-19 poses within each country, state, and health care environment evolves.

- This document is not intended as a guide for other clinical scenarios, epidemics, or pandemics.

\section{SHARED DECISION MAKING AND TRANSPARENCY}

Transparency regarding the potential risks of deferring or proceeding with an operation remains a priority. Surgeons should discuss these decisions individually with their patients. Multidisciplinary teams are encouraged to develop alternative treatment strategies if surgical resection is declined or infeasible.

\section{ORIGINS OF CONSENSUS STATEMENT}

This initiative is an extension of the American College of Surgeons and Commission on Cancer (CoC) effort to provide guidance for surgeons to make difficult triaging decisions in the face of progressively limited access to operating rooms, and there may be some slight differences in this document compared with the CoC-published documents. A partnership was formed between the $\mathrm{CoC}$ (Tim Mullett, Larry Shulman, Linda Martin, and Matt Facktor), the Thoracic Surgery Outcomes Research Network (ThORN, a research collective of board-certified general thoracic surgeons), and leaders from the American College of Surgeons (Heidi Nelson, Valerie Rusch, and Douglas Wood), and reviewed by leadership from The Society of Thoracic Surgeons and the American Association of Thoracic Surgery (David Jones and Shaf Keshavjee). The limited data were discussed in an open exchange, and the resulting guide is best characterized as being based on "expert opinion" in terms of strength of evidence. The authors recognize that multiple resources are becoming available to triage all types of surgical treatment. We intentionally avoided language that is currently being used to structure guidance based on procedures (ie, tiers) or patient status 
(ie, emergent, urgent, and semiurgent) to avoid confusion, and have instead organized recommendations based on the conditions that exist within each hospital ("phases").

\section{FINAL THOUGHT}

There are times when the right decision becomes easier-as the impact of the decision evaporates. This is one of those times. We hope that this document facilitates the timely execution of what are sure to be increasingly difficult decisions.

\section{APPENDIX OF CONTRIBUTING AUTHORS IN ALPHABETICAL ORDER}

Thoracic Surgery Outcomes Research Network, Inc: Mara Antonoff, MD, Leah Backhus, MD, Daniel J. Boffa, MD, Stephen R. Broderick, MD, Lisa M. Brown, MD, MAS, Phillip Carrott, MD, James M. Clark, MD, David Cooke, MD, Elizabeth David, MD, Matt Facktor, MD, Farhood Farjah, MD, MPH, Eric Grogan, MD, James Isbell, MD, David R. Jones, MD, Biniam Kidane, MD, Anthony W. Kim, MD, Shaf Keshavjee, MD, Seth Krantz, MD, Natalie Lui, MD, Linda Martin, MD, Robert A. Meguid, MD, MPH, Shari L. Meyerson, MD, Tim Mullett, MD, Heidi Nelson, MD, David D. Odell, MD, MPH, Joseph D. Phillips, MD, Varun Puri, MD, Valerie Rusch, MD, Lawrence Shulman, MD, Thomas K. Varghese, MD, Elliot Wakeam, MD, and Douglas E. Wood, MD.

\section{References}

1. Samson P, Patel A, Garrett T, et al. Effects of delayed surgical resection on shortterm and long-term outcomes in clinical stage I non-small cell lung cancer. Ann Thorac Surg. 2015;99:1906-12 [discussion: 1913].
2. Samson P, Crabtree TD, Robinson CG, et al. Defining the ideal time interval between planned induction therapy and surgery for stage IIIA non-small cell lung cancer. Ann Thorac Surg. 2017;103:1070-5.

3. Grotenhuis BA, van Hagen P, Wijnhoven BP, et al. Delay in diagnostic workup and treatment of esophageal cancer. J Gastrointest Surg. 2010;14: 476-83.

4. Kim JY, Correa AM, Vaporciyan AA, et al. Does the timing of esophagectomy after chemoradiation affect outcome? Ann Thorac Surg. 2012;93:207-12 [discussion: 212-3].

5. Liang W, Guan W, Chen R, et al. Cancer patients in SARS-CoV-2 infection: a nationwide analysis in China. Lancet Oncol. 2020;21:335-7.

6. Cao C, Wang D, Tian DH, et al. A systematic review and meta-analysis of stereotactic body radiation therapy for colorectal pulmonary metastases. J Thorac Dis. 2019;11:5187-98.

7. Rosen JE, Salazar MC, Wang Z, et al. Lobectomy versus stereotactic body radiotherapy in healthy patients with stage I lung cancer. J Thorac Cardiovasc Surg. 2016;152:44-54.e9.

8. Khorfan R, Kruser TJ, Coughlin JM, Bharat A, Bilimoria KY, Odell DD. Survival of primary stereotactic body radiation therapy compared with surgery for operable stage I/II non-small cell lung cancer. Ann Thorac Surg. 2020;110: 228-34.

9. Semenkovich TR, Meyers BF. Surveillance versus esophagectomy in esophageal cancer patients with a clinical complete response after induction chemoradiation. Ann Transl Med. 2018;6:81.

10. Levinsky NC, Wima K, Morris MC, Ahmad SA, Shah SA, Starnes SL, et al. Cincinnati Research in Outcomes and Safety in Surgery (CROSS) Group. Outcome of delayed versus timely esophagectomy after chemoradiation for esophageal adenocarcinoma. J Thorac Cardiovasc Surg. 2020;159:2555-66.

11. Bedford J, Enria D, Giesecke J, et al. COVID-19: towards controlling of a pandemic. Lancet. 2020;395:1015-8.

12. Li Q, Guan X, Wu P, et al. Early transmission dynamics in Wuhan, China, of novel coronavirus-infected pneumonia. N Engl J Med. 2020; 382:1199-207.

13. Guan WJ, Ni ZY, Hu Y, Liang WH, Ou CQ, He JX, et al; China Medical Treatment Expert Group for Covid-19. Clinical characteristics of coronavirus disease 2019 in China. N Engl J Med. 2020;382:1708-20.

14. Arentz M, Yim E, Klaff L, Lokhandwala S, Riedo FX, Chong M, et al. Characteristics and outcomes of 21 critically ill patients with COVID-19 in Washington State. JAMA. 2020;323:1612-4

15. Cabrini L, Landoni G, Zangrillo A. Minimise nosocomial spread of 2019-nCoV when treating acute respiratory failure. Lancet. 2020;395:685. 